\title{
O-tert-Butyltyrosine, an NMR Tag for High-Molecular-Weight Systems and Measurements of Submicromolar Ligand Binding Affinities
}

\author{
Wan-Na Chen, ${ }^{\dagger}$ Kekini Vahini Kuppan, ${ }^{\dagger}$ Michael David Lee, ${ }^{\ddagger}$ Kristaps Jaudzems, ${ }^{\S}$ Thomas Huber, ${ }^{* \dagger}$ \\ and Gottfried Otting ${ }^{*}, \dagger$
}

${ }^{\dagger}$ Research School of Chemistry, Australian National University, Canberra, ACT 2601, Australia

${ }^{\ddagger}$ Monash Institute of Pharmaceutical Sciences, Parkville, VIC 3052, Australia

${ }^{\S}$ Latvian Institute of Organic Synthesis, Riga 1006, Latvia

\section{Supporting Information}

\begin{abstract}
O-tert-Butyltyrosine (Tby) is an unnatural amino acid that can be site-specifically incorporated into proteins using established orthogonal aminoacyl-tRNA synthetase/tRNA systems. Here we show that the tert-butyl group presents an outstanding NMR tag that can readily be observed in one-dimensional ${ }^{1} \mathrm{H}$ NMR spectra without any isotope labeling. Owing to rapid bond rotations and the chemical equivalence of the protons of a solvent-exposed tert-butyl group from Tby, the singlet resonance from the tert-butyl group generates an easily detectable narrow signal in a spectral region with limited overlap with other methyl resonances. The potential of the tert-butyl ${ }^{1} \mathrm{H}$ NMR signal in protein research is illustrated by the observation and assignment of two resonances in the Bacillus stearothermophilus DnaB hexamer $(320 \mathrm{kDa})$, demonstrating that this protein preferentially assumes a 3-fold rather than 6-fold symmetry in solution, and by the quantitative measurement of the submicromolar dissociation constant $K_{\mathrm{d}}(0.2 \mu \mathrm{M})$ of the complex between glutamate and the Escherichia coli aspartate/ glutamate binding protein (DEBP, $32 \mathrm{kDa}$ ). The outstanding signal height of the ${ }^{1} \mathrm{H}$ NMR signal of the Tby tert-butyl group allows $K_{\mathrm{d}}$ measurements using less concentrated protein solutions than usual, providing access to $K_{\mathrm{d}}$ values 1 order of magnitude lower than established NMR methods that employ direct protein detection for $K_{d}$ measurements.
\end{abstract}

\section{INTRODUCTION}

NMR spectroscopy offers structural information on biological macromolecules at a level of detail that is unsurpassed by any other solution method. The resolution of the solution NMR spectrum of a large protein, however, is compromised both by the number of NMR resonances as well as by line widths that increase with molecular mass. ${ }^{1}$ These problems are routinely addressed by labeling with ${ }^{15} \mathrm{~N}$ and ${ }^{13} \mathrm{C}$ to facilitate the recording of multidimensional NMR spectra. In addition, perdeuteration of the protein leads to narrower NMR signals of the remaining ${ }^{1} \mathrm{H}$ spins, permitting in exceptional cases twodimensional (2D) NMR spectra to be recorded of protein multimers approaching total molecular masses of $500 \mathrm{kDa}$ and beyond. ${ }^{2-4}$ In particular, methyl groups are relatively easy to observe in such systems because methyl groups rotate rapidly, which results in chemical shift degeneracy of the three methyl protons and favorable ${ }^{1} \mathrm{H}$ relaxation properties. ${ }^{5}$ It is a quite general phenomenon that enhanced mobility produces narrower, more easily observable NMR signals. For example, highly flexible polypeptide segments are typically found at the $\mathrm{N}$ - and C-termini of a protein and their NMR resonances can often be observed without perdeuteration even when the molecular weight of the system is large. ${ }^{6}$

These observations prompted us to investigate the potential of an unnatural amino acid containing a mobile tert-butyl group.
Methods have recently been established for the genetic encoding of O-tert-butyltyrosine (Tby), ${ }^{7,8}$ but NMR spectra of proteins with Tby incorporated in this way have not been reported, although the value of monitoring the NMR signal of a site-specifically incorporated chemical "spy" is well established. For example, Geierstanger and co-workers have shown that the NMR resonances of the methyl- and $\mathrm{CF}_{3}$-groups of $p$ methoxyphenylalanine (OMePhe) and p-trifluoromethoxyphenylalanine $\left(\mathrm{OCF}_{3} \mathrm{Phe}\right)$ can readily be observed after sitespecific incorporation at different locations of a $33 \mathrm{kDa}$ protein. ${ }^{9}$ The signals of the newly introduced ${ }^{13} \mathrm{CH}_{3}$ - and $\mathrm{CF}_{3}$ groups were readily observed in two-dimensional (2D) ${ }^{13} \mathrm{C}$ HSQC and $1 D{ }^{19} \mathrm{~F}$ NMR spectra, respectively. Similarly, Mehl and co-workers demonstrated that site-specific incorporation of trifluoromethylphenylalanine $\left(\mathrm{CF}_{3} \mathrm{Phe}\right)$ produced $1 \mathrm{D}{ }^{19} \mathrm{~F}$ NMR spectra that allowed distinction of different ligand- and metalbound states in 48 and $98 \mathrm{kDa}$ homodimers. ${ }^{10}$ While the ${ }^{1} \mathrm{H}$ NMR resonances of methyl groups are difficult to resolve without additional labeling with ${ }^{13} \mathrm{C}$, the main drawback of ${ }^{19} \mathrm{~F}$ NMR is the large chemical shift anisotropy (CSA) associated with ${ }^{19} \mathrm{~F}$ spins which leads to broad NMR resonances. As CSA relaxation rates increase with the square of the magnetic field

Received: February 21, 2015

Published: March 19, 2015 
strength, ${ }^{19} \mathrm{~F}$ NMR spectra are best recorded at relatively low NMR frequencies, compromising sensitivity. In contrast, the tert-butyl group of Tby can be observed by ${ }^{1} \mathrm{H}$ NMR with increasing sensitivity at increasing magnetic fields. We hypothesized that, owing to the intensity of the methyl resonance (corresponding to nine degenerate ${ }^{1} \mathrm{H}$ spins) and the decreased relaxation rate associated with a mobile, solvent exposed tert-butyl group, its observation by high-field NMR should be straightforward without any stable isotope labeling.

In the following we show that Tby indeed produces a ${ }^{1} \mathrm{H}$ NMR signal that is readily observable in proteins without isotope labeling, including a $320 \mathrm{kDa}$ system. Furthermore, we show that the outstanding sensitivity of the tert-butyl resonance allows its detection in dilute protein solutions, effectively reducing the protein demand by 1 order of magnitude. Finally, we show that Tby is an excellent probe for direct NMR measurements of submicromolar ligand binding affinities.

\section{RESULTS}

Incorporation of Tby. For site-specific incorporation of Tby into proteins, we chose the $p$-cyanophenylalanyl-tRNA synthetase ( $p$ CNF-RS) evolved from the Methanocaldococcus jannaschii tyrosyl-RS encoded in the pUltra system together with the requisite $\mathrm{tRNA}_{\mathrm{CUA}}$ for recognition of TAG stop codons. ${ }^{11}$ Bacillus stearothermophilus (Bst) DnaB was produced in fusion with the solubility enhancer GB1 by in vivo expression in Escherichia coli (Supporting Information Figures S1 and S2A), whereas Staphylococcus aureus sortase A (SrtA) and the E. coli aspartate/glutamate binding protein (DEBP) were produced from linear PCR amplified DNA in a cell-free system based on an E. coli cell extract, from which the release factor 1 had been selectively removed by tagging with chitin-binding domains and filtration over chitin (Figure S2B). ${ }^{12}$ The fusion with GB1 did not significantly change the $1 \mathrm{D}{ }^{1} \mathrm{H}$ NMR spectrum of the Bst DnaB hexamer (Figure S3A).

NMR Spectroscopy of DnaB-Tby. Figure 1A compares the $1 \mathrm{D}{ }^{1} \mathrm{H}$ NMR spectrum of the mutant Tyr104Tby with the corresponding spectrum of wild-type DnaB. As expected for a high-molecular-weight system, the proteins display very broad signals, whereas very narrow signals arise from buffer or lowmolecular-weight impurities. Two new signals at about 1.2 ppm, however, are observed for DnaB-Tby but not wild-type DnaB. Their chemical shifts agree with the chemical shift of the tert-butyl group in free Tby (1.26 ppm in $25 \mathrm{mM}$ Tris- $\mathrm{HCl}, \mathrm{pH}$ 7.5). The observation of two resonances is in agreement with the two different chemical environments of the Tby site in crystal structures of the Bst DnaB hexamer in complex with DnaG (Figure 1B and C). ${ }^{13}$ Previous electron microscopy studies of E. coli DnaB had reported hexamers with 3-fold and 6-fold symmetries. ${ }^{14-16}$ The observation of two, rather than one, tert-butyl resonances identifies the 3-fold symmetry as the symmetry prevailing for the Bst helicase in solution.

To assign the local environments which the two resonances belong to, we introduced the additional mutations Cys253Val, Ala303Cys, and Cys305Val to enable site-specific ligation with a lanthanide tag at position 303. At the same time, the natural amino acids of the protein were perdeuterated to confirm the assignment of the tert-butyl signals. The mutations did not alter the appearance of the tert-butyl signals (Figure 1A). Following ligation of the cysteine residue with a $\mathrm{C} 1-\mathrm{tag}^{17}$ loaded with $\mathrm{Ce}^{3+}$ (Figure S4A), one of the two Tby resonances broadened significantly, assigning it to the site, which is within $15 \AA$ of $A l a$ 303 in the crystal structure. This result presents further
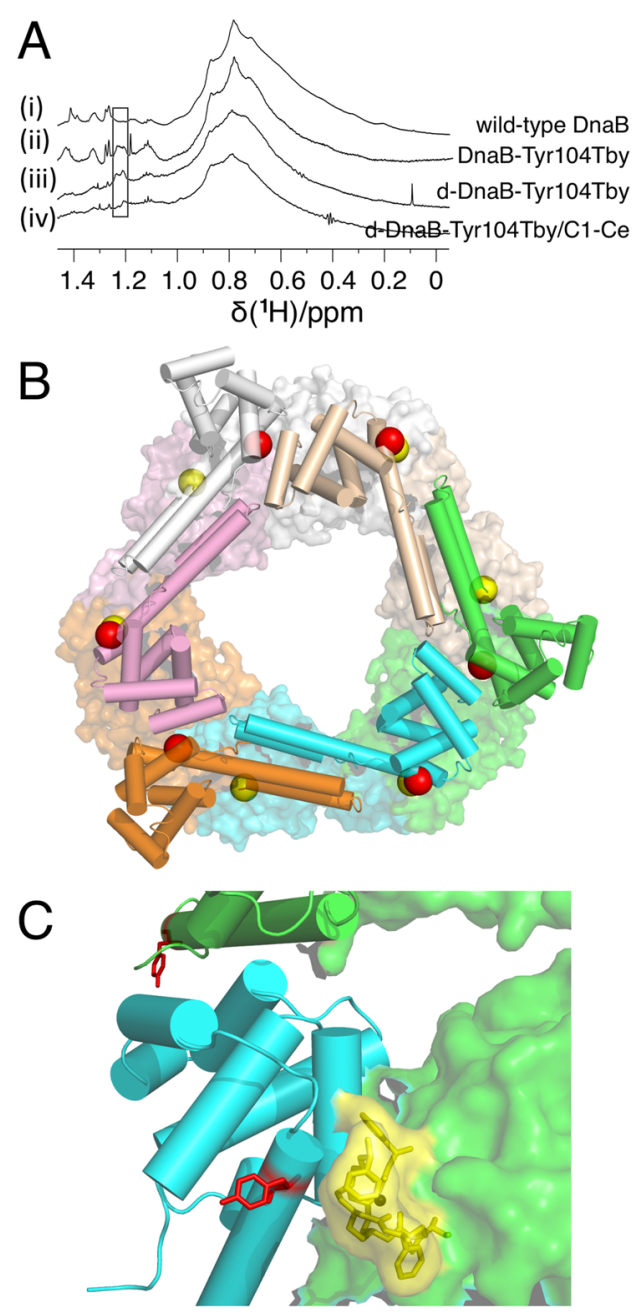

Figure 1. Conformational study of Bst DnaB helicase. (A) $1 \mathrm{D}{ }^{1} \mathrm{H}$ NMR spectra of wild-type and mutant Bst DnaB. The spectra were recorded in a buffer of $25 \mathrm{mM}$ Tris- $\mathrm{HCl}, \mathrm{pH} 7.5,150 \mathrm{mM} \mathrm{NaCl}$ at 25 ${ }^{\circ} \mathrm{C}$, using a Bruker $600 \mathrm{MHz}$ NMR spectrometer. Protein concentrations were about $20-40 \mu \mathrm{M}$ and the total recording time per spectrum was $0.5-2 \mathrm{~h}$. (i) Wild-type DnaB. (ii) DnaB with Tby at site 104. The tert-butyl group displays two peaks (highlighted by a frame). Unassigned narrow signals are from impurities in the buffer. (iii) Perdeuterated DnaB (70\% deuteration) with Tby at site 104 and containing the additional mutations Cys253Val, Ala303Cys, and Cys305Val. The reduced intensity of the background confirms the identification of the Tby tert-butyl resonances. (iv) Same as (iii), except with a C1-Ce tag ligated to Cys 303. The downfield, but not the upfield, tert-butyl peak is affected, assigning the downfield peak to the Tby residue near residue 303 of the neighboring DnaB molecule, and confirming that residue 104 exists in two different chemical environments as expected for a hexamer with C3 symmetry. (B) View of the crystal structure of the DnaB hexamer along the C3 symmetry axis (PDB: 2R6A). ${ }^{13}$ Each monomer is colored differently, showing the $\mathrm{N}$-terminal domains in a cartoon representation, while the linker segments and the C-terminal domains are in a surface representation. The sites of Tyr 104, which was replaced by Tby in this work, are highlighted by red spheres. The sites of residue 303, mutated to a cysteine for subsequent ligation with the $\mathrm{C} 1-\mathrm{Ce}$ tag, are identified by yellow spheres. (C) The C1-Ce tag modeled at Cys 303 (yellow) is close to Tyr 104 in the blue monomer, but far from Tyr 104 in the green monomer in the crystal structure 2R6A. The N- and $\mathrm{C}$-terminal domains are shown in cartoon and surface representations, respectively, as in (B). Tyr 104 is shown in red. 
confirmation that the crystal structure of the Bst hexamer reflects its predominant three-dimensional (3D) structure in solution.

Most importantly, the result clearly demonstrates the possibility to detect the Tby tert-butyl resonance in systems of high molecular mass at low concentration without any isotope labeling. For comparison, we also incorporated a trifluoromethyl-phenylalanine residue in position 104 and recorded a ${ }^{19} \mathrm{~F}$ NMR spectrum at a ${ }^{1} \mathrm{H}$ NMR frequency of $500 \mathrm{MHz}$. The line width of the ${ }^{19} \mathrm{~F}$ NMR resonance was about $160 \mathrm{~Hz}$ at half height and thus too broad to resolve two different signals (Figure S5).

NMR Spectroscopy of Sortase A with Tby. Figure $2 \mathrm{~A}$ and $B$ compares the $1 \mathrm{D}{ }^{1} \mathrm{H}$ NMR spectra obtained with the

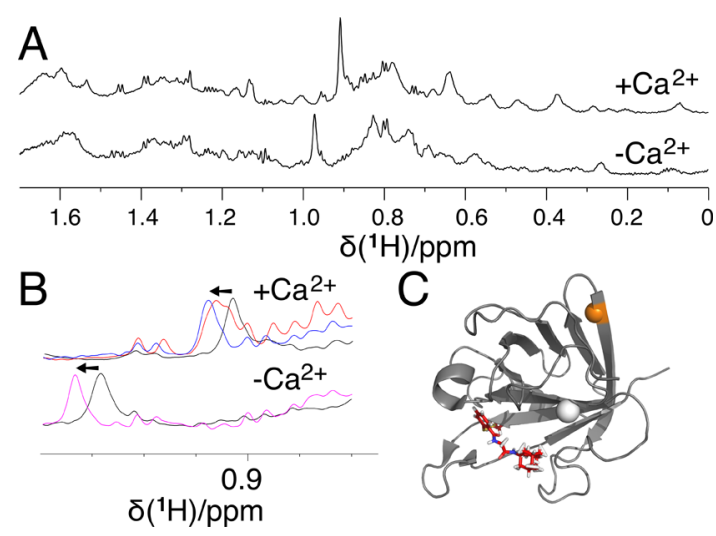

Figure 2. Ligand binding study of $S$. aureus sortase A. The spectra were recorded at $25{ }^{\circ} \mathrm{C}$ using a Bruker $800 \mathrm{MHz}$ NMR spectrometer. (A) $1 \mathrm{D}{ }^{1} \mathrm{H}$ NMR spectra of sortase A with Tby incorporated at position 150. Top trace: $20 \mu \mathrm{M}$ protein in $20 \mathrm{mM}$ MES buffer, $\mathrm{pH} 6.5,50 \mathrm{mM}$ $\mathrm{NaCl}, 5 \mathrm{mM} \mathrm{CaCl}$. Bottom trace: same as above, but without $\mathrm{CaCl}_{2}$ and with $1 \mathrm{mM}$ EDTA. (B) $1 \mathrm{D}{ }^{1} \mathrm{H}$ NMR spectra of a sortase A/ inhibitor complex with (top) and without (bottom) calcium. The protein concentration was $20 \mu \mathrm{M}$. Black: inhibitor-free state. Red: halfsaturation with inhibitor. Blue and magenta: inhibitor-bound state with $50 \%$ excess inhibitor. The inhibitor ${ }^{18}$ was added from a $5 \mathrm{mM}$ stock solution in DMSO- $d_{6}$. The final concentration of DMSO in the protein solution was always less than $1 \%$. Arrows indicate the direction of chemical shift change upon addition of inhibitor. (C) Cartoon representation of sortase A (PDB: $\left.1 \mathrm{IJA}^{19}\right)$. Inhibitor, calcium, and the mutation site for Tby are shown as red sticks, a white sphere, and an orange sphere, respectively. The ligand and calcium coordinates were taken from the PDB files $2 \mathrm{MLM}^{18}$ and $2 \mathrm{KID},{ }^{20}$ respectively.

Thr150Tby mutant of S. aureus sortase A and the effects of calcium and inhibitor binding on the chemical shift of the tertbutyl resonance of Tby. The chemical shift of the tert-butyl group is unusually high-field, which can be attributed to ring current effects arising from its proximity to the side chain of Phe 144. Despite the distance of the Tby residue from the active site marked by Cys 184 (about $25 \AA$ ) and the calcium binding site (about $20 \AA$, Figure $2 \mathrm{C}$ ), the chemical shift of the tert-butyl resonance was sensitive to the presence of $\mathrm{Ca}^{2+}$ or inhibitor, or both. Therefore, the Tby residue can be used for remote sensing of changes in the substrate binding site of the enzyme. Although the presence of calcium causes many spectral changes in the ${ }^{1} \mathrm{H}$ NMR spectrum of the protein, the tert-butyl resonance is easily picked out as the tallest resonance in the range between 0 and $1.7 \mathrm{ppm}$ (Figure 2A). The size of the resonance greatly facilitates monitoring its chemical shift in titration experiments, and its narrow line width magnifies the significance of even small chemical shift changes. The inhibitor used here (Figure S4B) is known to bind irreversibly by formation of a covalent bond to the active-site cysteine. ${ }^{18}$ As expected for a system with slow or no chemical exchange, the tert-butyl resonances of the inhibitor bound and unbound states of the protein appeared simultaneously at half-saturation (Figure 2B). A control experiment confirmed that the dimethyl sulfoxide (DMSO) of the inhibitor stock solution caused much smaller chemical shift changes than the ligand (Figure S3B).

Measuring Submicromolar Dissociation Constants. E. coli DEBP is a periplasmic transport protein that reversibly binds aspartate or glutamate. It binds glutamate with a binding affinity of $260 \mathrm{nM}$, as measured by isothermal calorimetry (ITC; B. Clifton and C. J. Jackson, personal communication). Despite the high molecular mass of the protein $(32 \mathrm{kDa})$, the tert-butyl resonance is clearly visible in the $1 \mathrm{D}{ }^{1} \mathrm{H}$ NMR spectrum of the mutant Val140Tby and over 1 order of magnitude taller than any other signal of the protein (Figure 3A). Therefore, NMR spectra of good sensitivity can be

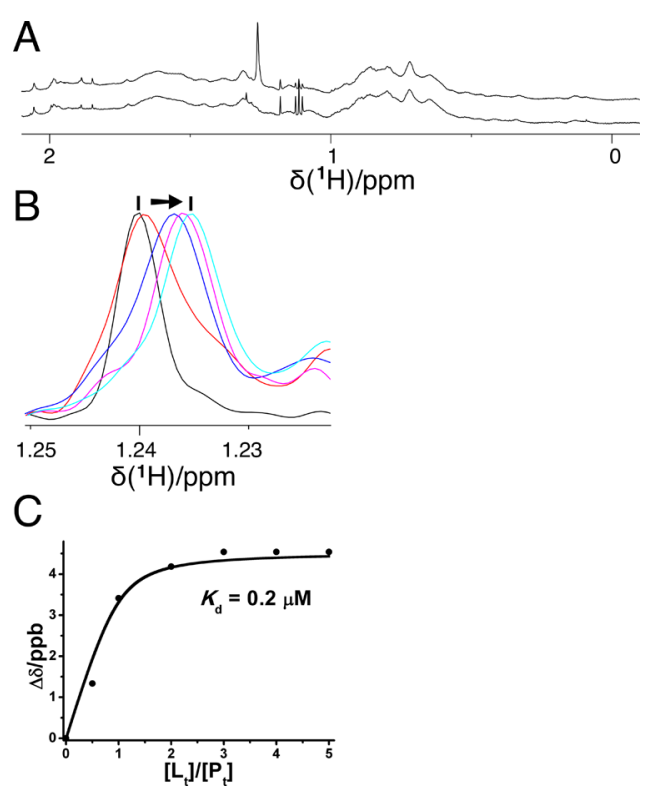

Figure 3. Binding affinity measurement of E. coli DEBP for glutamate. The 1D ${ }^{1} \mathrm{H}$ NMR spectra were recorded in $20 \mathrm{mM}$ phosphate buffer, $\mathrm{pH} 7.5,100 \mathrm{mM} \mathrm{NaCl}$ at $25{ }^{\circ} \mathrm{C}$, using a Bruker $800 \mathrm{MHz} \mathrm{NMR}$ spectrometer. (A) NMR spectra of $50 \mu \mathrm{M}$ solutions of DEBP. The top and bottom traces show the spectra of the mutant with Tby at position 140 and of wild-type DEBP, respectively. The signal of the tert-butyl group appears at about $1.24 \mathrm{ppm}$. Other narrow signals are from lowmolecular weight impurities. (B) Titration of glutamate $(1 \mathrm{mM}$ stock solution in $\mathrm{H}_{2} \mathrm{O}$ ) into a $2 \mu \mathrm{M}$ solution of DEBP. The tert-butyl resonance shifts upfield with increasing concentration of glutamate (indicated by the arrow). Each spectrum was acquired with a total recording time of $30 \mathrm{~min}$ on a $800 \mathrm{MHz}$ NMR spectrometer equipped with a cryoprobe. (C) Fit of the binding affinity curve by eq 1 . For best accuracy, the chemical shifts were determined by fitting Lorentzian line shapes to the tert-butyl resonances in (B). $K_{\mathrm{d}}$ was calculated as 0.20 $\mu \mathrm{M}$ with a standard error of $0.08 \mu \mathrm{M}$.

recorded at low protein concentrations, which is a prerequisite for determining small dissociation constants from chemical shift changes of protein resonances. Titration of glutamate into the protein solution indeed led to small changes in chemical shifts, indicating fast exchange between the bound and free state of the protein (Figure 3B). 

1 :

The observed chemical shift changes can be described by eq

$$
\begin{aligned}
\Delta_{\text {obs }}= & \Delta_{\max }\left\{\left(K_{\mathrm{d}}+L_{\mathrm{t}}+P_{\mathrm{t}}\right)\right. \\
& \left.-\left[\left(K_{\mathrm{d}}+L_{\mathrm{t}}+P_{\mathrm{t}}\right)^{2}-4 P_{\mathrm{t}} L_{\mathrm{t}}\right]^{1 / 2}\right\} /\left(2 P_{\mathrm{t}}\right)
\end{aligned}
$$

where $\Delta_{\text {obs }}$ is the observed change in chemical shift, $\Delta_{\max }$ is the maximal chemical shift change observed when the protein is fully saturated with ligand, $L_{\mathrm{t}}$ and $P_{\mathrm{t}}$ are the total concentrations of ligand and protein, respectively, and $K_{\mathrm{d}}$ is the dissociation constant. The $K_{\mathrm{d}}$ value obtained by fitting the observed changes in chemical shifts by eq 1 was $0.20 \mu \mathrm{M}$ (Figure 3C), in close agreement with the value determined by ITC measurement.

Near-Isotropic Bond Reorientation in Tby. The tertbutyl group of Tby produces an exceptionally narrow line because of its motional freedom. When it is fully solvent exposed, three different bond rotations contribute to the reorientation of the $\mathrm{C}-\mathrm{H}$ bonds of the methyl groups. First, each methyl group rotates about its axis of symmetry. Second, the tert-butyl group reorientates by rotation about the $\mathrm{C}-\mathrm{O}$ bond, that projects from the aromatic ring. Finally, the following $\mathrm{O}-\mathrm{C}$ bond to the tert-butyl group is free to rotate. Crystal structures of compounds with tert-butyl-phenyl groups show dihedral angles of about $90^{\circ}$ for the $\mathrm{C}-\mathrm{O}$ bond, positioning the tert-butyl group outside the plane of the aromatic ring (Figure 4A). Assuming dihedral angles of $90^{\circ}$ or
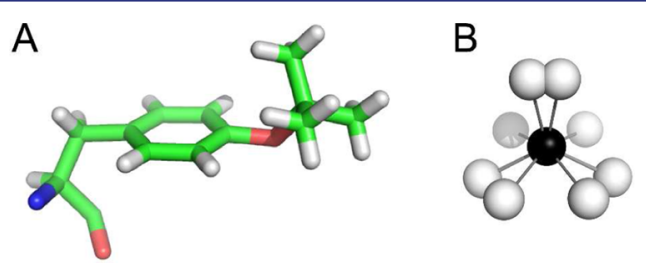

Figure 4. Methyl groups of O-tert-butyl-tyrosine can change their orientation in multiple directions. (A) Stick representation of the $\mathrm{O}$ tert-butyl-tyrosine residue as determined by X-ray crystallography. ${ }^{21}$ (B) Different orientations of a single methyl $\mathrm{C}-\mathrm{H}$ bond produced by rotations around bonds of the O-tert-butyl group, including dihedral angles of $90^{\circ}$ or $270^{\circ}$ for the $\mathrm{C}-\mathrm{O}$ bond, and $60^{\circ},-60^{\circ}$, or $180^{\circ}$ for the $\mathrm{O}-\mathrm{C}$ bond and the methyl groups. The variability in the resulting $\mathrm{C}-\mathrm{H}$ bond orientations is highlighted by superimposing the carbon atom of each of the 18 rotameric states. The carbon and hydrogen atoms of the $\mathrm{C}-\mathrm{H}$ group are represented by black and white spheres, respectively. Some of the rotamers produce degenerate orientations.

$270^{\circ}$ for the $\mathrm{C}-\mathrm{O}$ bond, and $60^{\circ},-60^{\circ}$, or $180^{\circ}$ for the $\mathrm{O}-\mathrm{C}$ bond and the methyl group rotamers, an individual $\mathrm{C}-\mathrm{H}$ group of a methyl can access $2 \times 3 \times 3=18$ different rotameric states. Assuming equal probability of each dihedral angle combination owing to unrestricted motions of a solvent-exposed tert-butyl group, the $\mathrm{C}-\mathrm{H}$ bond populates a remarkably isotropic distribution of orientations (Figure 4B). As a measure of the degree of isotropy, concatenation of all $\mathrm{C}-\mathrm{H}$ bond vectors produced by the 18 rotameric states yields a sum vector of a length of only about $1 \AA$. Although each rotamer may be associated with different opportunities for dipole-dipole relaxation depending on the location of other protein protons in the vicinity, the remarkably slow relaxation of the tert-butyl resonance is fundamentally due to its exceptionally high degree of mobility.

\section{DISCUSSION}

The present work shows that tert-butyl-tyrosine is an outstanding probe for the study of small and large proteins at natural isotopic abundance by NMR spectroscopy. The intense ${ }^{1} \mathrm{H}$ NMR signal of the tert-butyl group is easily detected in onedimensional NMR spectra due to its narrow line width and chemical shift that is well separated from the NMR resonances of most organic buffers, the water resonance, and the usually dominant methyl signals of valine, leucine, and isoleucine. The system for genetic encoding of Tby is well established, ${ }^{7}$ allowing its incorporation at any site of a protein. Importantly, the ${ }^{1} \mathrm{H}$ chemical shift of the tert-butyl resonance is still sensitive to changes in the chemical environment of the protein, even if the tert-butyl group is solvent exposed. Therefore, it can be used to monitor the state of a protein, which is of particular interest for studies of ligand binding.

Measurements of Dissociation Constants. The results obtained with DEBP demonstrate the outstanding advantage of Tby for ligand binding studies, in particular for the measurement of tight binding affinities. Measurements of small dissociation constants by direct observation of protein signals require a protein concentration in the order of the $K_{d}$ value. The intensity of the tert-butyl resonance provides the sensitivity required to measure dilute solutions even of large proteins.

A graphical representation of titration curves for different $K_{d}$ values illustrates the difficulty to measure dissociation constants that are much smaller than the protein concentration (Figure 5 ). To obtain $K_{\mathrm{d}}$ values with reasonable accuracy, a practical

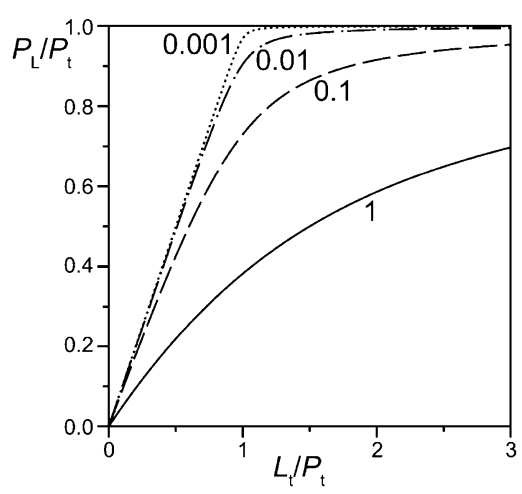

Figure 5. Titration curves calculated for different dissociation constants $K_{\mathrm{d}}$. The curves were calculated using eq 1 . The vertical axis plots the saturation of the protein with ligand (ratio of bound protein, $P_{\mathrm{L}}$, relative to total protein, $P_{\mathrm{t}}$ ). The horizontal axis plots the ratio of total ligand concentration, $L_{t}$, to total protein concentration. The curves are labeled with the respective dissociation constants normalized by the total protein concentration, $K_{\mathrm{d}} / P_{\mathrm{t}}$.

recommendation is to aim for protein concentrations that produce saturation ratios of ligand-bound protein to total protein between 0.2 and 0.8 , when ligand and protein are present in 1:1 ratio. ${ }^{22}$ Figure 5 shows that this condition is fulfilled for $K_{\mathrm{d}}$ values that are 10 times smaller than the protein concentration, but not if they are 100 or 1000 times smaller (see also Figure S6). Therefore, the measurement of submicromolar $K_{d}$ values requires the ability to measure at protein concentrations in the low micromolar range. Such low concentrations pose a serious challenge in sensitivity for contemporary NMR spectrometers, especially if the NMR resonances of the protein are broad due to high molecular weight or chemical exchange broadening. As a result, NMR 
spectroscopy is traditionally regarded as a unique tool for detecting complexes with weakly binding ligands, whereas $K_{\mathrm{d}}$ values much smaller than about $10 \mu \mathrm{M}$ are practically inaccessible by direct observation of protein resonances even in the case of small proteins, ${ }^{23}$ let alone proteins of the molecular mass of DEBP. The exceptional peak height of the ${ }^{1} \mathrm{H}$ NMR resonance of the tert-butyl group of Tby changes this situation and lowers the detection limit by more than an order of magnitude. In principle, ligand detected competition experiments are a viable alternative for determining nanomolar binding affinities by NMR spectroscopy, but these experiments are less straightforward than the direct monitoring of an NMR resonance of the target protein in a protein observed experiment because they require the availability of an additional, different ligand, for which the dissociation constant must be determined independently. ${ }^{24}$

Tight binding affinities are invariably associated with slow dissociation rates, as the dissociation constant can be calculated from the ratio of dissociation versus association rates and diffusion poses an upper limit on the rate of complex formation. In the limit of slow chemical exchange between bound and free protein, separate NMR signals are observed and eq 1 no longer applies. Importantly, however, the limit between the fast and slow chemical exchange regimes depends on the frequency difference of the NMR resonances in the respective states, with small frequency differences extending the fast exchange regime to slower exchange rates. For the measurement of small dissociation constants, it is thus an advantage if the NMR signal which is monitored displays only small chemical shift changes upon titration with ligand, as in the case of a solvent-exposed tert-butyl group. As the examples of sortase A and DEBP show, the narrow line shape of the ${ }^{1} \mathrm{H}$ NMR resonance of the tertbutyl group of Tby permits accurate measurements also of small changes in chemical shift, allowing the positioning of the Tby probe sufficiently far from the ligand binding site to avoid any interference with ligand binding. In practice, we found that narrow NMR signals from buffer or low-molecular weight impurities provide excellent internal controls to confirm the accurate alignment of the NMR spectra recorded in a titration experiment.

Tby as a Probe in High-Molecular-Weight Systems. Established strategies for solution NMR spectroscopy of highmolecular weight systems employ isotope labeling strategies, in particular per-deuteration to reduce dipolar relaxation rates of the remaining ${ }^{1} \mathrm{H}$ spins. ${ }^{25,26}$ Selective labeling or unlabeling of all amino acid residues of a certain type, however, usually labels more than a single site, making it challenging to obtain sitespecific information. In contrast, the incorporation of a single unnatural amino acid naturally produces a label at a predetermined site, which is defined by the amber stop codon placed in the gene of interest.

In the case of Bst DnaB, the Tby probe positioned at a solvent exposed site of the $\mathrm{N}$-terminal domain of the protein not only revealed clear ${ }^{1} \mathrm{H}$ NMR resonances of the tert-butyl group, but also provided clear evidence for the $\mathrm{C} 3$ symmetry of the hexamer as two different chemical environments of the tertbutyl group were apparent. Considering the molecular mass of the hexamer $(320 \mathrm{kDa})$, it is remarkable that this could be achieved without isotope labeling, highlighting the substantial line narrowing associated with a mobile tert-butyl group of Tby. Once a site-specific signal can be observed, it offers a convenient probe for further structural analysis. In the case of Bst DnaB we were able to verify the crystal structure of the hexamer by the selective line broadening observed for one of the signals, when an intermonomer contact with a paramagnetic tag was produced in a cysteine mutant of the protein. The C1tag is a cyclen-based lanthanide-binding tag designed to induce pseudocontact shifts (PCS). In the case of the Bst DnaB mutant with Tby residue and $\mathrm{C} 1-\mathrm{Ce}$ tag, the observation of line broadening rather than PCS can be attributed to paramagnetic relaxation enhancement, which becomes more severe with increasing molecular weight, ${ }^{27}$ or to reduced motional freedom of the tert-butyl group due to interactions with the tag. For protein systems of lower molecular mass, where the tert-butyl signal is more readily observable, it will be possible to use a lanthanide tag to gain further structural information by inducing pseudocontact shifts in the tert-butyl resonances of Tby residues positioned at different sites of the protein.

\section{CONCLUSION}

In conclusion, the Tby probe offers superior sensitivity in ${ }^{1} \mathrm{H}$ NMR spectra, which makes larger and less soluble proteins amenable to NMR analysis than hitherto possible. The NMR signal of the tert-butyl group can be observed with minimal interference from the protein background. The robustness with which the tert-butyl group can be detected also in the presence of large concentrations of buffer and ligands could be enhanced further by ${ }^{13} \mathrm{C}$ labeling. While, in principle, isotope labels can be deployed to study wild-type proteins, the introduction of a Tby residue requires a point mutation. Notably, however, the capacity to study proteins without perdeuteration is important too, as perdeuterated proteins can be significantly less stable than their unlabeled forms, which can result in slightly different structures. $^{28,29}$ Unlabeled Tby is commercially available and inexpensive. We anticipate that the Tby probe will become an extremely valuable tool in structural biology and drug discovery.

\section{ASSOCIATED CONTENT}

\section{S Supporting Information}

Figures illustrating the DnaB construct used, the production of full-length versus truncated protein, the effect of cleaving GB1 from DnaB, the effect of DMSO on tert-butyl group chemical shifts, the chemical structures of the C1-Ce tag and the sortase A inhibitor, $1 \mathrm{D}{ }^{19} \mathrm{~F}$ NMR spectra of trifluoromethyl-phenylalanine labeled DnaB, a glutamate binding affinity measurement performed with $10 \mu \mathrm{M}$ DEBP, and the nucleotide sequence of the construct of E. coli DEBP. This material is available free of charge via the Internet at http://pubs.acs.org.

\section{AUTHOR INFORMATION}

\section{Corresponding Authors}

*gottfried.otting@anu.edu.au

*t.huber@anu.edu.au

\section{Notes}

The authors declare no competing financial interest.

\section{ACKNOWLEDGMENTS}

We thank Prof. Nicholas E. Dixon for helpful discussions on DnaB, Prof. Peter G. Schultz for the pEVOL and pUltra vector with the $p$-cyano-Phe-tRNA synthetase, Dr. Bim Graham for the C1-Ce tag, and Dr. Colin J. Jackson for an expression system of wild-type DEBP. W.-N.C. thanks the government of the People's Republic of China for a CSC scholarship. Financial support by the Australian Research Council is gratefully acknowledged. 


\section{REFERENCES}

(1) Ryabov, Y. E.; Geraghty, C.; Varshney, A.; Fushman, D. J. Am. Chem. Soc. 2006, 128, 15432-15444.

(2) Fiaux, J.; Bertelsen, E. B.; Horwich, A. L.; Wüthrich, K. Nature 2002, 418, 207-211.

(3) Sprangers, R.; Kay, L. E. Nature 2007, 445, 618-622.

(4) Mas, G.; Crublet, E.; Hamelin, O.; Gans, P.; Boisbouvier, J. J. Biomol. NMR 2013, 57, 251-262.

(5) Rosenzweig, R.; Kay, L. E. Annu. Rev. Biochem. 2014, 83, 291315.

(6) Ozawa, K.; Horan, N. P.; Robinson, A.; Yagi, H.; Hill, F. R.; Jergic, S.; Xu, Z.-Q.; Loscha, K. V.; Li, N.; Tehei, M.; Oakley, A. J.; Otting, G.; Huber, T.; Dixon, N. E. Nucleic Acids Res. 2013, 41, 53545367.

(7) Young, D. D.; Young, T. S.; Jahnz, M.; Ahmad, I.; Spraggon, G.; Schultz, P. G. Biochemistry 2011, 50, 1894-1900.

(8) Wang, Y.-S.; Fang, X.; Wallace, A. L.; Wu, B.; Liu, W. R. J. Am. Chem. Soc. 2012, 134, 2950-2953.

(9) Cellitti, S. E.; Jones, D. H.; Lagpacan, L.; Hao, X.; Zhang, Q.; Hu, H.; Brittain, S. M.; Brinker, A.; Caldwell, J.; Bursulaya, B.; Spraggon, G.; Brock, A.; Ryu, Y.; Uno, T.; Schultz, P. G.; Geierstanger, B. H. J. Am. Chem. Soc. 2008, 130, 9268-9281.

(10) Jackson, J. C.; Hammill, J. T.; Mehl, R. A. J. Am. Chem. Soc. 2007, 129, 1160-1166.

(11) Chatterjee, A.; Sun, S. B.; Furman, J. L.; Xiao, H.; Schultz, P. G. Biochemistry 2013, 52, 1828-1837.

(12) Loscha, K. V.; Herlt, A. J.; Qi, R.; Huber, T.; Ozawa, K.; Otting, G. Angew. Chem., Int. Ed. 2012, 51, 2243-2246.

(13) Bailey, S.; Eliason, W. K.; Steitz, T. A. Science 2007, 318, 459463.

(14) San Martin, M. C.; Stamford, N. P. J.; Dammerova, N.; Dixon, N. E.; Carazo, J.-M. J. Struct. Biol. 1995, 114, 167-176.

(15) Yu, X.; Jezewska, M. J.; Bujalowski, W.; Egelman, E. H. J. Mol. Biol. 1996, 259, 7-14.

(16) San Martin, C.; Radermacher, M.; Wolpensinger, B.; Engel, A.; Miles, C. S.; Dixon, N. E.; Carazo, J.-M. Structure 1998, 6, 501-509.

(17) Graham, B.; Loh, C. T.; Swarbrick, J. D.; Ung, P.; Shin, J.; Yagi, H.; Jia, X.; Chhabra, S.; Pintacuda, G.; Huber, T.; Otting, G. Bioconjugate Chem. 2011, 22, 2118-2125.

(18) Zhulenkovs, D.; Rudevica, Z.; Jaudzems, K.; Turks, M.; Leonchiks, A. Bioorg. Med. Chem. 2014, 22, 5988-6003.

(19) Ilangovan, U.; Ton-That, H.; Iwahara, J.; Schneewind, O.; Clubb, R. T. Proc. Natl. Acad. Sci. U. S. A. 2001, 98, 6056-6061.

(20) Suree, N.; Liew, C. K.; Villareal, V. A.; Thieu, W.; Fadeev, E. A.; Clemens, J. J.; Jung, M. E.; Clubb, R. T. J. Biol. Chem. 2009, 284, 24465-24477.

(21) Goldup, S. M.; Pilkington, C. J.; White, A. J. P.; Burton, A.; Barrett, A. G. M. J. Org. Chem. 2006, 71, 6185-6191.

(22) Fielding, L. Prog. NMR Spectrosc. 2007, 51, 219-242.

(23) Marti, D. N.; Hu, C.-K.; An, S. S. A.; von Haller, P.; Schaller, J.; Llinás, M. Biochemistry 1997, 36, 11591-11604.

(24) Dalvit, C.; Fasolini, M.; Flocco, M.; Knapp, S.; Pevarello, P.; Veronesi, M. J. Med. Chem. 2002, 45, 2610-2614.

(25) Gardner, K. H.; Kay, L. E. Annu. Rev. Biophys. Biomol. Struct. 1998, 27, 357-406.

(26) Lu, K.; Miyazaki, Y.; Summers, M. F. J. Biomol. NMR 2010, 46, $113-125$.

(27) Otting, G. J. Biomol. NMR 2008, 42, 1-9.

(28) Hattori, H.; Crespi, H. L.; Katz, J. J. Biochemistry 1965, 4, 12131225.

(29) Piszczek, G.; Lee, J. C.; Tjandra, N.; Lee, C.-R.; Seok, Y.-J.; Levine, R. L.; Peterkofsky, A. Arch. Biochem. Biophys. 2011, 507, 332342 . 